\title{
Italique
}

Poésie italienne de la Renaissance

XX | 2017

Varia

\section{Prima esplorazione di una linea di ricerca: la bucolica come veicolo di tradizione indiretta}

\section{Claudio Vela}

\section{(2) OpenEdition}

1 Journals

\section{Edizione digitale}

URL: http://journals.openedition.org/italique/461

DOI: 10.4000/italique.461

ISSN: $1663-4438$

\section{Editore}

Librairie Droz

\section{Edizione cartacea}

Data di pubblicazione: 1 ottobre 2017

Paginazione: 173-193

ISBN: 978-2-600-05818-6

ISSN: 1423-3983

Notizia bibliografica digitale

Claudio Vela, «Prima esplorazione di una linea di ricerca: la bucolica come veicolo di tradizione indiretta », Italique [Online], XX | 2017, online dal 01 octobre 2019, consultato il 23 janvier 2020. URL: http://journals.openedition.org/italique/461; DOI : 10.4000/italique.461 


$$
\text { CL A U D I O VE A }
$$

PRIMA ES PLORAZIONE D I U A LINEA D I RICERCA: LA B UCOLICA COME VEICOLO D I TRA DIZIONE IN DIRET T A 



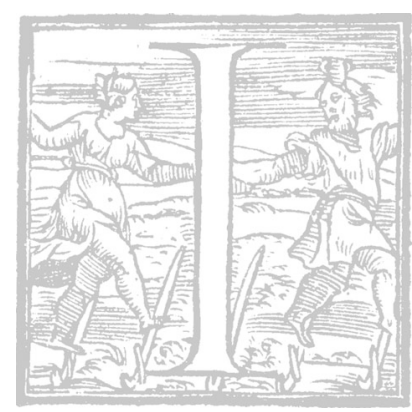

I fenomeno che voglio prendere in considerazione non è specifico della poesia bucolica, non ne costituisce un elemento strutturale esclusivo. Ma poiché si può manifestare anche nella bucolica, e storicamente vi si è manifestato, non importa quanto marginalmente o sporadicamente, mi è sembrato che potesse essere fatto oggetto di qualche indagine dedicata, che almeno una prima esplorazione, come suggerisce il titolo del mio intervento per doverosa correttezza (davvero solo di una prima esplorazione si tratta), potesse essere tentata.

Tanto più che l'argomento, anzi meglio l'indagine, si è venuta quasi d'obbligo a porre all'intersezione di alcune diverse linee delle mie ricerche: la tradizione indiretta nel suo filone principale per quanto riguarda $i$ testi letterari italiani, dunque soprattutto i 'trattati grammaticali' (per usare un'etichetta imperfetta ma comoda) come, per stare piu direttamente ai miei studi, le Prose della volgar lingua del Bembo; la poesia per musica tra Quattro e Cinquecento con la sua ricchezza di citazioni interne; e soprattutto, l'unica reale incursione che mi è capitato di fare nel territorio della letteratura pastorale, con lo studio e l'edizione dell'egloga Tirsi di Baldassar Castiglione e Cesare Gonzaga (nellontano 1998). ' Testoparticolare proprio per l'argomento qui proposto: infatti entro i 454 versi che lo compongono (5s stanze inframmezzate da una 'canzonetta' in forma di ballata) 4 versi (e una parte di un quinto) raccolgono esplicitamente versi altrui, intendendo con altrui non solo "di altri autor" (cosa che vale sicuramente almeno per alcuni di essi, ma si discute se per tutti), ma certamente "da altri testi". Lo status di citazione di queste parti del testo è evidente, come appare subito alla lettura.

Si tratta delle stanze 40-4I e 43-44 (quattro su cinquantacinque):

Dal sino d'Hadria qua venne un pastore

Fra tutti gli altri assai famoso e degno,

Qual sentendo di questa el gran valore

Solo a cantar di lei pose el suo inzegno;

Et ha del suo splendor sì vago el core,

Che non curò lassare il patrio regno,

Ma venne ad habitar questo paese

E cantò dolcemente: Alma cortese. 
Venne dal Mintio quel che al secol nostro

Via più cresce l'honor, cresce la fama:

Questo è sì noto nel paese vostro,

Che ogni pastor di là l'honora et ama;

So c'hai veduto del suo sacro inchiostro

Là ove si duol d'amore e mercé chiama.

Dolce e amaro destin, che mi sospinse,

Cantò l'altr'hieri, e tutti gli altri vinse.

$[\ldots]$

Venne d'Hetruria un altro in questi monti

Saggio e docto pastore in ciascuna arte;

Non son piaggie qui atorno o rive o fonti,

Che non intendan le sue lode sparte.

Ma temo assai che prima el sol tramonti,

Ch'io possa dir di lui pur una parte.

Questo cantò con amorosa voce:

Se fosse el passo mio cosi veloce.

Stassi tra questi anchor un giovinetto

Pastor, che a dir di lui pietate prendo:

Così fu grave il duol, grave il dispetto

Che già gli fece amor, sì come intendo,

Ch'egli ne porta anchor piagato el petto;

E mille fiate al dì si duol dicendo:

Io son forzato, Amore, a dire hor cose

A te di poco honore, a me noiose.

Mi sembra interessante in primo luogo verificare le condizioni di status letterario che hanno permesso agli autori del Tirsi di inserire (o invogliato a inserire) queste citazioni. E cioè: si tratta di una innovazione o di un motivo tradizionale? ci sono modelli diretti e/o indiretti? Chiaramente si intrecceranno qui due ordini di considerazioni: da una parte quello generale della tradizione indiretta nelle sue articolazioni (esiste una articolazione 'bucolica' della tradizione indiretta? e quando si presenta e come si sviluppa?), dall' altra quello specifico del Tirsi, che, salvo errore (ma appunto da qui parte una esplorazione che io ho condotto solo per campione), sembra il testo pastorale (ma siamo al limite del genere) più ricco di un simile artificio. Perché? A questa 
domanda proverò a dare una risposta in conclusione. Ora vorrei concentrarmi invece sul primo punto, cioè provare a indicare possibili modelli o semplicemente precedenti di una tale consuetudine citatoria (e dunque di tradizione indiretta dei testi citati, peraltro coi limiti e problemi, proprio di natura testuale, che si vedranno).

In realtà, il primo modello che viene in mente non è un testo bucolico, ma una canzone che appartiene a un testo la cui immanenza sulla poesia anche primocinquecentesca non può essere sottovalutata né tanto meno ignorata: ed è ovviamente Lasso me, ch'i' non so in qual parte pieghi, la canzone 70 dei Rerum vulgarium fragmenta, la canzone delle citazioni appunto, dei versus cum auctoritate. La situazione è tutt' altra rispetto a quella del Tirsi, basti dire che i versi altrui e il proprio citati da Petrarca vengono tutti rifunzionalizzati, per cosi dire, all'interno del nuovo testo, e neppure si dice che appartengono ad altri poeti (naturalmente il primo caso proclama da sé la propria diversa appartenenza, essendo in lingua d'oc, unica occorrenza in tutto il canzoniere di Petrarca, che lo credeva di Arnaut Danie), non vengono cioè presentati come citazioni. ${ }^{3}$ Ma dalla canzone 70 è ben probabile che derivi la collocazione esplicitaria di strofa (di ottava nel caso del Tirsi) della citazione, il cui testo viene dunque assunto nel sistema delle rime (allo stesso modo di Petrarca). Anche la variazione introdotta nella stanza 4I, in cui il verso altrui occupa non l'ultimo ma il penultimo posto della stanza, non infrange questa struttura di base. Come non la infrangono ma la valorizzano le altre variazioni che gli autori si concedono: del primo testo, quello del Bembo, evidentemente già proverbiale, non è necessario riportare tutto l'incipit ma basta il primo emistichio (Alma cortese), di un altro, quello finale (dunque è una sorta di cornice di variazione quella che qui viene costruita), si danno addirittura due versi, che sono non incipitari ma conclusivi ( $v v$. IO-II) della prima stanza della canzone Perché di lachrimar son stanco homai (tràdita adespota dal Magl. VII 727 della Naz̧ionale di Firenze, testimone unico). ${ }^{4} \mathrm{Ma}$ la differenza più spiccata sta nel fatto che in Petrarca $i$ citati stanno nell' $\ll$ olimpo dei poeti passati》:.5 ed è questo anzi che 'adegua' e assimila per cosi dire Petrarca, l'unico vivente, allo (pseudo-)Arnaut Daniel, e a Cavalcanti, Dante e Cino da Pistoia (la datazione relativa della canzone è offerta esattamente dal termine a quo della morte di Cino, I336). Mentre nel Tirsi tutti $i$ citati, Bembo (autore di Alma cortese, la canzone in morte del 
fratello Carlo) e Giuliano de' Medici (autore del sonetto Se fosse il passo mio così veloce), ${ }^{6}$ e gli altri due, chiunque essi siano, sono contemporanei agli autori, agiscono nello stesso ordine di valori poetici e nello stesso tempo e spazio, cosi tenuemente adombrato dalla finzione pastorale, della corte urbinate del primo decennio del Cinquecento. Gli studiosi e i commentatori di Petrarca, in primo luogo Santagata e la Bettarini, hanno mostrato la trafila tradizionale, in particolare medioevale, che sta dietro l'artificio citatorio della canzone 70, che la riassume innovandola, e dunque la annulla per chi viene dopo, stante anche l'immediato statuto modellizzante dei Rvf. La innova, come nota la Bettarini, soprattutto "nell'ultima stanza dell'autocitazione, ritornando a Virgilio; il quale con geniale semplicità cita un suo verso-titolo a chiusura non solo di componimento o di libro, ma di un'intera vicenda poetica giovanile, quando sigilla le Georgiche evocando il primo verso della prima Egloga, "Tytire, te patulae cecini sub tegmine fagi", come indubbiamente fa qui Petrarca, richiamando quel dolce tempo dal quale comincia la sua frammentata storia di poeta d'amores. ${ }^{7}$ I precedenti medievali 'barbarici' potevano essere bellamente ignorati, meno facilmente invece, data la presenza pervasiva di Virgilio tra $i$ poeti anche in volgare del primo Cinquecento, quella chiusa cosi particolare delle Georgiche.

Le quali fungono per noi da tramite, vista proprio la sostanza dell'autocitazione, esattamente verso l'Arcimodello di questa poesia, le Bucoliche di Virgilio. Ora, si può discutere sullo status di 'tradizione indiretta' dell'autocitazione, ma sta di fatto che anche l'autocitazione consiste nell'assunzione di un frammento di un testo all'interno di un testo diverso: almeno una doppia indicazione se ne ricava, dato che gli elementi sono due, non uno: di 'esistenza in vita' (almeno presupposta) dell'intero testo di cui si cita un per quanto piccolo estratto (molto spesso l'incipit, ma non solo), e della sua collocazione cronologica relativa rispetto al testo ospitante. Ma oltre a questo è evidente che la presenza di una simile trovata, che vorrei definire di 'autopoetica', nel Virgilio delle Georgiche, non può non spingerci nella stessa direzione di ricerca entro le Bucoliche. E magari da lì risalire più su, verso l'ulteriore modello dietro Virgilio, verso l'inventore stesso del genere, Teocrito. Insomma mi sembra legittimo ricercare le 'autorizzazioni' classiche del fenomeno (non distinguendo, anche se bisognerebbe farlo, tra citazione e autocitazione, visto che ciò che importa è la rilevazione 
tout court di una tradizione indiretta autorizzata, articolata come si voglia, entro il genere bucolico).

In Teocrito, e stiamo pure al solo Teocrito attivo in Virgilio, ${ }^{8}$ nell'idillio IV (I pastori) Coridone offre «un campione più realistico delle sue capacità anzitutto con l'elogio di Crotone, citando verosimilmente solo l'esordio della composizione, (che dunque prende le mosse dal confronto con Zacinto [...])», cioè «Bella città è sia Zacinto...», v. 32. ${ }^{9}$ Ma meglio ancora nell'idillio VII (Le Talisie, molto influente su Virgilio), in cui ai vv. 50-53 Licida dice "Quanto a me - vedi, amico, se ti piace questa canzoncina che di recente elaborai sul monte: "Avrà Ageanatte una felice navigazione verso Mitilene" $):$ un canto nel canto. ${ }^{\circ}$ Più direttamente coinvolto nel fenomeno l'idillio XIV (Eschina e Tionico), dove al v. 30 Eschina rivela: "Ebbene, già noi quattro eravamo sprofondati nella bevuta, quando il Larisseo attaccò: "Il mio Lupo", un motivo tessalo». Commenta Vox: "Agide di Larissa, città tessala, attacca una canzone delle sue parti, forse nota dalle prime parole (una citazione analoga in id. IV 32). Richiamando Orazio, Odi I 32, 3 ss. W. Beck ipotizza che si tratti in realtà dell'attacco (misconosciuto da Eschina) di un poema omoerotico di Alceo dedicato a Lico (l'aria sarebbe detta tessalica per l'affinità fra parlate eolica e tessala)». ${ }^{\text {I }}$ Quest'ultimo, soprattutto se ha ragione il Beck, sarebbe un vero caso di tradizione indiretta. Ma non dimentichiamoci, perché tra poco dovremo occuparcene più diffusamente, dell'altra interpretazione, che si tratti invece di «una canzone delle sue parti, forse nota dalle prime parole» (con tanto di rinvio a idillio $\mathrm{IV}, 32)$. Dove la citazione riguarderebbe testi musicati, 'canzoni' nella terminologia attuale.

Con Teocrito alle spalle (perlomeno fino allidillio VIII compreso), Virgilio fa nelle Bucoliche qualcosa di un po' diverso. Inaugura infatti qui la pratica dell'autocitazione effettiva (intendo, ripresa letterale, o tenuemente adattata al nuovo contesto, ad es. per adeguamento metrico, di un segmento di un testo veramente preesistente), e le affianca anche una modalità, molto più controversa, di cui però parlerò poi trattando dell'Arcadia. L'autocitazione è quella, doppia, dell'egloga V, 85-87. Dice Menalcas-Virgilio:

Hac te nos fragili donabimus ante cicuta; haec nos «formosum Corydon ardebat Alexin», haec eadem docuit «cuium pecus? an Meliboei?». 
Si tratta degli incipit di Ecl. II (Formosum pastor Corydon ardebat Alexin) e III (Dic mihi, Damoeta, cuium pecus? an Meliboei?), il primo con la soppressione di pastor, il secondo più scorciato. Ma ciò che importa è che in questo modo Virgilio autorizza a citare se stessi, anch'egli confermando la consuetudine di identificare opere letterarie citandone l'incipit a mo' di titolo (si può effettivamente parlare di incipit-titolo). ${ }^{12}$

Allora ecco che trovano un appoggio anche esterno, non di mero artificio interno, $i$ casi emblematici dell'Arzocchi e del Boiardo. Ci sono voluti gli studi del secondo Novecento per dare fisionomia e importanza, un'importanza perfino decisiva per le sorti della bucolica volgare, al primo, il senese Francesco Arzocchi, le cui egloghe godono oggi, fortunatamente e giustamente, di un'edizione, critica e commentata. ${ }^{13}$ Non ci sono dubbi per quanto riguarda il v. I63 dell'egl. II, detto dall'anonimo pastore (che rappresenta l'autore):

e questo fu già chiosa in un mio testo con latte dello fico disegnato, all'occhio bruno che m'è sì molesto dicendo: Io sono il cervio innaverato che non sa metter la sua pena a' rughi.

Caso decisamente interessante di tradizione indiretta, assimilabile, nella differenza, alla citazione dell'incipit della canzone dantesca Tragemi de la mente Amor la stiva nel De vulgari eloquentia. Perché come di quello dantesco anche di questo testo dell' Arrocchi nulla conosciamo se non le parole citate: è poco, ma non pochissimo: puntello testuale per futuri eventuali riconoscimenti del testo in tradizione diretta e per la sua attribuibilità, ma anche, positivamente, per l'attestazione del raro innaverato ('ferito'), parola duecentesca qui in testimonianza preziosa, e c'è da chiedersi per quali tramiti sia arrivata all'Arzocchi, $e$ in sede di rima. ${ }^{\mathrm{I}}$ Meno perspicui $i$ casi di I, 28 e soprattutto di III, 68-72, perché che si tratti di autocitazioni è una probabile ipotesi, ma non una certezza. Il primo sta nella richiesta di Grisaldo-Arzocchi all'interlocutore Terinto: ${ }^{\mathrm{IS}}$

- Deh, or dimmi quella pur Come la nottola, che tu l'altr'ier cantavi sotto un suvaro!

- Dunque m'udisti? Or, ella è una frottola. 
Dicevo non cosi perspicuo perché mentre in II, I63 il pastore-autore parla esplicitamente di «un mio testo» qui la 'copertura bucolica' che attribuisce a Terinto lo specifico canto può riferirsi tanto all' autore-Arzocchi quanto al suo interlocutore; dipiù, può trattarsi di mera escogitazione a posteriori, non di autocitazione o citazione, e dunque non di tradizione indiretta. Lo stesso vale, in modo ancor più complicato, per III, 67-72: ${ }^{16}$
lassando lui dolente che diceva:
«Chi si dispera mai non giugne tempo».
Ohimè, come presso a me l'aveva!
Ed io partimmi rimpiangendo il tempo
ch'io avevo perduto per vaghezza,
dicendo: «Male aspetta chi ha tempo».

La Fornasiero nota intelligentemente che questi versi «sembrano alludere a qualcosa di più personale - e noto ai destinatari primi - come una corrispondenza poetica di cui sarebbero dunque allegati qui i versi iniziali». Ipotesi «difficilmente sostenibile», come lei stessa ammette, «in mancanza di documenti». ${ }^{17}$ Ma è bene che l'ipotesi, non incompatibile col codice bucolico, anzi, sia stata fatta; è bene che ci abituiamo a incasellare versi o parti di verso che potrebbero essere citazioni nel repertorio virtuale dei frammenti di tradizione indiretta. Un impreveduto ritrovamento non è escluso che possa trasferire quel virtuale in un reale. Meglio dunque una inclusione ipotetica, perfino indebita, che una esclusione virtualmente smentibile dai fatti. Proprio perché lo statuto del genere, già nei modelli Teocrito e Virgilio, contempla la possibilità dell'autocitazione e, per estensione, della citazione, non è implausibile aspettarsele, per quanto minoritario il fenomeno possa essere.

Clamoroso invece, e inequivocabile, e come tale già più volte 'preso in conto' negli studi, il fenomeno dell'autocitazione in Boiardo, nei noti versi di Pastorale V, 25-26: ${ }^{\mathrm{I}}$

E' mi ramenta già che de to' versi alquanti ne sapeva, et hor mi dole che eccetto questi dua tuti l'ho persi «Amor che me scaldava al suo bel sole nel dolcie tempo di mia età fiorita». Più non ne sciò, che scorse ho le parole. 
Come già fatto dall'Arzochi in I, 28 (e sulla base della comune autorizzazione virgiliana di Ecl. V), che come è ormai acquisito costituisce per Boiardo un modello insieme da imitare e emulare, anche Boiardo inserisce nell'egloga addirittura due versi (uno solo, e non completo, in Arzocchi) che confermano volutamente l'intonazione lirica della prima parte dell'egloga, perché sono i versi incipitari degli Amorum libri, il canzoniere lirico del Boiardo. ${ }^{\text {I9 }}$ Secondo Zanato nel suo commento agli Amorum Libri questi versi sono citati «espressamente come incipit di riconoscimento degli AL nella Pastorale». ${ }^{20}$ (E se ne ricava, aggiungo tra parentesi per l'ovvietà dell'osservazione, anche una direzione cronologica, che conferma l'egloga $V$ come posteriore agli Amorum libri, o almeno al sonetto iniziale). E va notato come in questo modo Boiardo ottenga un ulteriore effetto: certificare che Menalca è lui stesso, darsi una patente di autoattriburione. ${ }^{2 \mathrm{I}}$

Ma in realtà non è questo l'unico caso di tradizione indiretta nella produzione pastorale in Boiardo, pur che facciamo un diverso passo e uscendo dalle egloghe volgari interroghiamo quelle latine. Qui infatti troviamo uno spunto interessante non solo in sé ma particolarmente fecondo di altri richiami, che ci permetteranno poi un aggancio anche con l'Arcadia di Sannazaro. Mi riferisco ai vv. 25-27 dell'egloga ИI dei Pastoralia, detti dal pastore Poeman a Coridone: ${ }^{22}$

Tu tibi forte aliquid magnum cecinisse videris cum «Rosa bella» tuo consurgit carmine, vel cum proditur a pueris iam cognitus «Ille triumphus».

E stata rilevata la comunanza della doppia citazione in questa e nell'egloga III di Tito Vespasiano Strozzi. ${ }^{23}$ Dunque anche nell'egloga latina era possibile citare composizioni musicali di normale circolazione quattrocentesca; e c'entra anche la cronologia: si tratta di un Boiardo giovane, due decenni prima delle Pastorale volgari. Ma questo ci porta in un territorio cosi particolare quale quello dei rapporti tra poesia e musica, soprattutto nella sua declinazione 'popolare' (con tutte le virgolette del caso), della quale non poche volte abbiamo una conoscenza solo riflessa, appunto attraverso citazioni di titoli o di versi in tradizione indiretta. $^{24}$ Ovviamente non è una caratteristica della sola bucolica, anzi. Ma anche in questo genere il fenomeno è attestato, e merita più attenzione di quanto finora non gli sia stata riservata. Basti pensare, per esempio, al diverso trattamento riservato finora dagli studi a due 
testi entrambi richi di citazioni di questo tipo. Il primo è l'opera generale di Teofilo Folengo, entro la quale si colloca anche la versione maccheronica dell'egloga, la Zanitonella. Ebbene, uno studio specifico di Giulio Cattin del 1975 è andato a identificare e a commentare tutte le numerose citazioni di balli o canti o composizioni musicali presenti in Folengo: la parte del leone la fa naturalmente il Baldus, ma ecco che anche nella Zanitonella troviamo menzione di Titalora, della Tortorella, del Matarello e della Pavana, della Rosina e di un bellissimo "Para foras, belle Gianole, capras», che porta nel latino maccheronico «O Zano, bello Zano, caza fora le capre». ${ }^{25}$ La stessa attenzione non è stata riservata invece all'egloga rusticale del notaio bolognese Cesare Nappi, spesso citata quando ci si occupa di poesia per musica, ma per la quale siamo ancora all'edizione del Frati sul «Giornale Storico della Letteratura Italiana» del I892. Entro i 364 versi (I2I terzine + verso finale) dell'egloga, i vv. I72-86, nella finzione - rusticale più che pastorale - sono detti da Borro, in un contesto in cui si balla e si invita al ballo: ${ }^{26}$

Ballato ho in ferrarese, anche in Verona,

In Bologna e so far la Fiorentina,

La Fontanella e Coza over Rangona,

La Pigna, el Pegoraro e Coregina,

La Piva, el Turlurù col[lo] Spingardò,

El Tortion, la Bissa e Ramacina,

E Tiente alora e 'l Piridun far so:

Ancora E me livava un bel mattino,

Va in là, poltron, va tuò el to bo,

El Saltarello e ancora el bel Gerbino,

Sciò far la Turturina e Sargia e ancora

De Levate la strenga el ballettino.

Torala mo villan quel fo d'ogn'hora;

Tosa to mar te chiama è sol mio ballo;

Fortuna d'un gran tempo tegno un'hora.

In quindici versi vengono citati ventiquattro balli, riferendone quasi sempre il titolo ma almeno una volta ("Va in là, poltron, va tuò el to bo») un intero verso (per quanto ipometro, ma basterebbe replicare "va in là» dopo «poltron»), certamente incipitario. ${ }^{27}$

Nella radicale diversità che intercorre tra la nostra epoca e l'inizio del 
Cinquecento non si può dire che la struttura del fatto sia poi tanto cambiata: un elenco di successi, la citazione di titoli e incipit che immediatamente dicevano qualcosa ai lettori/spettatori/ascoltatori, riportavano alla memoria musiche e parole, o balli.

Queste sopravvivenze di pura tradizione indiretta sono per noi oggi preziose, tanto più quando non ci resti altro. Si trattava di un repertorio abbastanza vasto, $i$ cui componenti ricostruiamo mettendo insieme liste come questa del Nappi a menzioni in altri autori e generi, come appunto quelle del Baldus oppure quelle teatrali, ad es., per citare il caso forse più famoso, nella Betìa del Ruzante, dove Nale nell' atto II ai vv. 429-4I cita $i$ titoli o gli incipit di Is «canzon». ${ }^{28}$ Anche se le fonti più ricche e importanti restano nei libri di frottole (nel senso musicale della parola) stampati da Ottaviano Petrucci tra il Iso4 e il I5I4. Via via che gli studi li affrontano col necessario approccio scientifico (per ora è stata procurata l'edizione critica, spesso arricchita da commento o altri sussidi, di 6 libri) ci si dispiega la riccherza di queste stampe che uniscono testi e musiche, e si conferma la ricchezza anche della tradizione indiretta, soprattutto di canti e composizioni 'popolari', di cui sono portatrici. ${ }^{29} \mathrm{E}$ il fatto che siano riportate in questo modo testimonia che si trattava di composizioni 'di successo', che si supponevano immediatamente riconoscibili dal pubblico a cui questo repertorio si rivolgeva.

Non stupirà allora di trovarne traccia, menzione forse di canto reale entro la finzione generale del canto, nell'Arcadia del Sannazaro. Dico forse perché non è arbitrario immaginare, data la sottigliezza e la suprema abilità costruttiva del Sannazaro, che questi citati siano anch'essi di invenzione dell'autore, in una finzione 'di secondo grado'. Sospetto dato dal fatto di non averli reperiti altrove. Ma ecco il passo, $v v$. 75-80 dell'egloga II (Itene all'ombra degli ameni faggi), dove inizia a parlare Montano: ${ }^{30}$

Or qual canterò io, che n'ho ben cento?

Quella del fier tormento?

O quella che comincia: Alma mia bella?

Dirò quell'altra forse: Ahi cruda stella?

(URANIO

Deh, per mio amor, di' quella

ch'a mezzo dì l'altrier cantasti in villa.) 
Vecce nel suo commento ricorda che qui si tratta di «Incipit convenzionali di canzonette per musica del Tre-Quattrocento», e inoltre che «il sintagma Abi cruda stella si trova anche nelle Rime di Filenio Gallo (26.6) e, variato in "cruda e dispietata stella", anche nelle Rime del De Iennaro (8.8)", oltre a rimandare anche allo schema di $\mathrm{Rvf} 70$ e alla conclusione della quinta giornata del Decameron, dove Dioneo propone una serie di 'canzoni' licenziose citando l'incipit (o i primi due versi) di alcune (ne ricorda nove, noi conosciamo il testo di una sola, Questo mio nicchio). ${ }^{3 \mathrm{I}}$ Il punto è esattamente questo: si tratta qui di tradizione indiretta, testi effettivamente esistenti, non diversamente da quelli riportati dal Nappi e dal Folengo, oppure, si diceva, di invenzione 'interna'? Fra l'altro anche da questi pochi sintagmi, che gli editori riportano in corsivo sottolineando dunque il carattere di citazione, emerge il carattere lirico, non rustico ("fier tormento», "alma bella», «cruda stella»). Senza contare che anche questa modalità si appoggia ancora una volta su Virgilio e, quanto alla bucolica volgare, al 'capostipite' Arzocchi, come si è visto.

Anticipavo che Virgilio oltre all'autocitazione presenta nelle Bucoliche una diversa, ma controversa modalità di inserzione di versi da altro testo, cosa che ha un diretto riflesso anche nell'Arcadia, per non uscire dal perimetro dell'opera sannazariana. Non mi riferisco al normale 'gioco di diffrazione' per cui un pastore riferisce il canto di un altro pastore, espediente pienamente inserito nel codice della tradizione bucolica (ad es. in Ecl. 2 e nell'egloga III dell'Arcadia), e che non può certo mai porsi, nella sua costruita finzione, come caso di tradizione indiretta. Mi riferisco invece alla discussa 'parte' di Cornelio Gallo nella X e ultima delle Bucoliche, dove per 39 versi (vv. 3I-69) Virgilio dà la parola a un personaggio identificato col suo nome reale, più volte ribadito. A partire da Servio si discute se alcuni di questi versi non siano di Virgilio ma effettivamente siano citazioni di composizioni di Gallo stesso ("hi autem omnes versus Galli sunt de ipsius translati carminibus», annota Servio al v. 46). ${ }^{32}$ Qui il codice bucolico permetterebbe di 'bucare la finzione' per cosi dire, cioè di inserire versi realmente altrui nella finzione di un autore che scrive ovviamente lui $i$ versi che invece si dicono composti e cantati da un altro autore. Ma come accertarlo, e come riconoscerli? e come verificare, in assenza di una tradizione diretta degli stessi versi, se questi non siano stati manipolati, adattati al nuovo contenitore? Per ora, sono domande senza risposta. 
E sono le stesse però che sorgono spontanee davanti al caso particolarissimo dell'egloga $x$ dell'Arcadia, l'ultima della prima redazione (il Libro pastorale nominato Arcadio). Dove i vv. 49-185, riportati da Selvaggio, si dicono però composti da "Caracciolo", cioè da Giovan Francesco Caracciolo, il cui nome, unico, non è coperto dal travestimento onomastico pastorale, visto che ricorre esplicitamente come "Caracciolo» al v. $4 I^{33}$ Il testo a lui attribuito viene incorniciato, com'è peraltro normale, da forti marche liminari: v. 47 «cosi prese a cantar sotto un bel frassino», e v. I86 «Cosi cantava, e i boschi rintonavano». ${ }^{34}$ Questo luogo poetico va messo in relazione con due passi della successiva prosa XI, rispettivamente ai commi I e 7. Dice il primo, iniziale:

Se le lunghe rime di Fronimo e di Selvaggio porsono universalmente diletto a ciascuno de la nostra brigata, non è da dimandare.

Ed è vero, perché sono Fronimo e Selvaggio a dire anche $i$ versi di Caracciolo. Ma specifica il comma 7:

E sopra tutto mi piacque udirla comendare [la patria, Napoli] de' studii de la eloquenzia e de la divina altezza de la poesia, e tra le altre cose de le merite lode del mio virtuosissimo Caracciolo, non picciola gloria de le volgari Muse; la canzone del quale, e se per lo coverto parlare fu poco da noi intesa, non rimase però che con attenzione grandissima non fusse da ciascuno ascoltata. ${ }^{35}$

È un passo la cui particolarità e importanza per l'interpretazione dell'intera Arcadia non è sfuggita agli studiosi. ${ }^{36}$ Io vorrei solo sottolineare come qui, caso unico salvo errore, si ribadisca ancora l'attribuzione del testo a un altro autore, un poeta vivente al momento della prima redazione dell'opera (Caracciolo era certamente vivente nel I498), e citato di nuovo col suo proprio nome: quasi come una chiosa insistente e chiarificatrice. Commenta Vecce: «A distanza di qualche anno, Sannazaro suggerisce che l'allegoria politica dell'egloga di Caracciolo non fu compresa dai contemporanei, e anzi interpretata in modi diversi (un fenomeno ricorrente nella ricezione della poesia cortigiana)». ${ }^{37}$ Ora, il fatto che alcuni di questi versi (83, II5, II7, I20-I23, I55, I59, I60, I83, I84) siano variati nel passaggio redazionale dell'Arcadia dimostrerebbe senza ombra di dubbio che essi non possano essere che di Sannazaro stesso, che qui fa fare un ulteriore salto di livello alla finzione. ${ }^{38}$ 
Ma non riesco a immaginare che il Caracciolo non ne sia stato, come minimo, informato; e consenziente; e perché non anche collaborante? In altre parole: è proprio illegittimo e arbitrario pensare che qui dentro ci siano uno o più riferimenti a testi effettivi del Caracciolo, testi che non ci sono noti per altra via? Forse anche Sannazaro poteva credere che entro la sua egloga finale davvero Virgilio avesse dato ospitalità a qualche effettivo verso di Gallo: e che nella sua propria il rinvio fosse a quel modello è dimostrato in modo certo dai vv. 47-48, immediati antecedenti del "Canto di Caracciolo" detto da Selvaggio: «cosi prese a cantar sotto un bel frassino / io fiscelle tessendo, egli una gabbia». ${ }^{39} \mathrm{E}$ in Virgilio, stavolta al termine del canto di Gallo, il poeta che dice "io", l'autore dunque, dice di sé "dum sedet et gracili fiscellam texit bibisco» (Ecl. $x$ 7I). Gallo-Caracciolo entro Virgilio-Sannazaro: due doppi perturbanti, perfino nella distribuzione della responsabilità testuale.

Ma è tempo di concludere e di tornare al Tirsi per provare a rispondere, dopo il frammentato percorso compiuto, alla domanda lasciata in sospeso, sulle ragioni di questa presenza di citazioni di altri testi. Un fenomeno che abbiamo potuto vedere non essere esclusivo del Tirsi, ma che altrove si presenta con altre modalità e con frequenza ridotta. Ma dobbiamo considerare che il Tirsi è un prodotto di corte per la corte, quella urbinate di Elisabetta Gonzaga, e rientra a pieno titolo nella letteratura 'feltresca'. Nella seconda parte del testo, quella più scopertamente celebrativa, per quindici ottave (33-47) Dameta tesse l'elogio della Duchessa (la «dea»), delle sue «ninfe» (le dame della corte) e dei "pastori» (letterati o musici) che la onorano. La finzione pastorale si fa esile: si nomina esplicitamente l'ambiente urbinate, «intorno al vago e bel Metauro»; una trasparente allusione rende omaggio all'amica più stretta di Elisabetta, Emilia Pio; infine si dà conto dei «pastori» che vengono da altri luoghi («externi»). Le ottave centrali, che ho citato allinizio, ospitano dunque una sorta di piccolo catalogo che si affianca a quello celebre e più vasto del Cortegiano nel presentare l'assetto culturale della corte urbinate all'altezza del I507-I508, introducendo $i$ personaggi che la frequentavano con citazioni da loro componimenti e/o riferimenti a particolari biografici. Mi pare evidente che si tratti qui di una sorta di 'poesia di cerchia', di 'poesia di amicizia', e che le citazioni presuppongano l'immediato riconoscimento della paternità dei testi: si tratti cioè di tradizione indiretta di una produzione immediatamente 
coeva, circolante in quel tempo e in quello spazio, riconoscibile alla corte di Urbino nel carnevale del I508 (una data che bo ricavato, contro la vulgata del I506, proprio grazie alla menzione di Alma cortese del Bembo, sicuramente composta non prima degli ultimi mesi del I507, e dunque freschissima novita). ${ }^{40}$ Cosa che da una parte, a mio parere, esclude che siano degli autori stessi, Castiglione e Gonzaga, $i$ due testi di attriburione dubbia, e dall'altra parte richiede che $i$ loro autori siano personaggi coerenti con lo spazio e col tempo della rappresentazione: nulla di più probabile che fossero presenti personalmente alla recitazione del testo davanti alla duchessa. Il pastore che «venne dal Mintio», un mantovano dunque, autore di Dolce e amaro destin che mi sospinse (un capitolo ternario che nella tradizione si trova o adespoto o erroneamente attribuito a Bembo), potrebbe essere Giovanni Muzzarelli, ricordato nella seconda redazione del Cortegiano (se fosse Castiglione si sarebbe presentato con una lode di se stesso cosi smaccata?). Mentre l'autore della coppia di versi finali di stanza di canzone (Perché di lachrimar son stanco homai), per cui si è proposto lo stesso Cesare Gonzaga (ma la canz. come si diceva è adespota nel Magl. VII 727, testimone unico), è più plausibile che sia Gasparo Pallavicino: "giovinetto» si addice molto meglio a uno nato nel I486, come il Pallavicino, che a un nato nel I475, come il Gonzaga; e il fatto che sia presentato come vittima di amore è coerente col suo personaggio nel Cortegiano, dove gli tocca la parte del misogino. ${ }^{4 \mathrm{I}}$

Qui dunque la tradizione indiretta davvero aumenta la nostra informazione, è un 'valore aggiunto', non solo o non tanto riguardo ai testi cosi testimoniati, ma perché ci permette, oltre che, come si è detto, di collocare nella giusta cronologia il testo ospitante (cosa non da poco, per le conseguenze che implica, in primo luogo il rapporto con le Stanze del Bembo), di ricostruire e capire piu fondatamente un momento di storia letteraria, in un preciso luogo e tempo. Il "codice bucolico" ha in questo caso per così dire fornito l'innesco. Il risultato è una ulteriore dimostrazione di come la filologia in definitiva altro non sia che 'intelligenza della storia'.

Claudio Vela 
I. C. Vela, Il «Tirsi» di Baldassar Castiglione e Cesare Gonzaga, in La poesia pastorale del Rinascimento, a cura di S. Carrai, Padova, Antenore, I998, pp. 245 -92.

2. Il testo, qui come nel seguito, è ripreso dalla mia edizione entro lo studio cit. alla nota precedente, pp. 282-83. Il corsivo è mio e segnala le citazioni da altri testi. Una più recente edizione del Tirsi in Baldassarre Castiglione - Cesare GonZaGa, "Rime" e "Tirsi", a cura di G. Vagni, Bologna, Emil di Odoya, 2015 , pp. I 87-254 (le ottave in questione sono alle pp. 238-39 e 24I-43).

3. Non così nel Tirsi, dove le 'marche di citazione' sono molto chiare, con presentazione del «pastore» in incipit e verbum dicendi (o meglio canendi) in explicit o verso l'explicit dell'ottava: 40, I e 8 «venne un pastore» «E cantò»; 4I, I e 8 «Venne dal Mintio quel» «Cantò l'altr'hieri»; 43, I e 7 «Venne d'Hetruria un altro» «Questo cantò»; 44, I e 6 «Stassi tra questi ancor un giovinetto» «si duol dicendo».

4. La canzone è pubblicata da Vagni tra le Rime dubbie di Cesare Gonzaga, in Baldassarre Castiglione - Cesare Gonzaga, "Rime" e "Tirsi", pp. i47-54; ma cfr. qui la nota $4 \mathrm{I}$.

5. Sono parole di Rosanna Bettarini, nel suo commento alla canzone petrarchesca: cfr. Francesco Petrarca, Canzoniere. "Rerum vulgariun fragmenta», a cura di R. Bettarini, Torino, Einaudi, 2005 , p. 346.

6. Cfr. il commento di Vagni in Baldassare Castiglione - Cesare Gonzaga, "Rime" e "Tirsi", p. 242.

\section{Francesco Petrarca, Canzoniere. «Rerum vulgarium fragmenta», p. 346.}

8. Cfr. su questo la voce Teocrito di Gregorio Serrao in Enciclopedia Virgiliana, Roma, Istituto della Enciclopedia Italiana, v*, I 990, pp. i Io- 8.

9. Cfr. Carmi di Teocrito e dei poeti bucolici greci minori, a cura di O. Vox, Torino, UTET, I997, p. I40 (testo in greco e commento, da cui la citazione a testo) e p. I4I (traduzione a testo).

Io. Carmi di Teocrito e dei poeti bucolici greci minori, p. I73. E cfr. il commento di Vox, p. 173 nota I 3: «canti nel canto di Licida già a sua volta interno, con un meccanismo sofisticato di 'scatole cinesi'». Vox rimanda a questo proposito al volume, che non ho visto, di S. Goldhill, The poet's voice. Essays on poetics and Greek literature, Cambridge, Cambridge U.P., I991, p. 233 ss.

I I. Carmi di Teocrito e dei poeti bucolici greci minori, p. 23 I, traduzione e commento. Il rimando a Beck si riferisce a W. A. Beck, Theocritus, Idyll I4: Alcaeus and Megara, in «Würzburger Jahrbücher für die Altertumswissenschaft», N. F. I8, 1992, pp. I 7 I -82 .

I 2. Una consuetudine rilevata dai commenti: cfr. ad es. quello a Ecl. v 86-87 di W. Clausen, A commentary on Virgil Eclogues, Oxford, Clarendon Press, I994, p. 172 : «The Second and Third Eclogues are identified by partial quotations of the first lines. Literary works were sometimes identified in antiquity by the opening words of the text: thus 'Utinam ne in nemore', Cic. De fin. r. 5; 'O Tite, si quid', Cic. $A d$ 
Att. I6.3.I; 'Aeneadum genetrix', Ov. Trist. 2.26I; 'Cynthia', Prop. 2.24.2, Mart. I4.I 89. I; 'Arma virumque', Pers. I.96, Mart. 8.55.19, I 4.1 8 5.2.).

i 3. Francesco Arzocchi, Egloghe, testo critico e commentato a cura di S. Fornasiero, Bologna, Commissione per i Testi di lingua, I995. I versi citati nel testo sono a p. 27.

I4. Nel suo commento, ivi, pp. 33-34, la Fornasiero osserva che la citazione «dovrebbe essere, per la logica dei rinvii intertestuali [...], l'incipit di un componimento lirico del pastore-autore, ma non se ne è trovata traccia. La recensio delle attestazioni del raro innaverato ('ferito') propone, come unico caso avvicinabile, Mare amoroso 275-8, dove cerbio e inaverato compaiono entrambi in posizione rilevata». Altre occorrenze duecentesche sono ora raccolte nel TLIO (alle voci innaverato e naverato), tra le quali una (innavrato) nel son. 2 I o di Guittone d'Arezzo (ediz. Egidi). Si aggiunga che la lezione innaverato è della Giuntina di rime antiche del i 527 al v. 3 del son. Deh piacciavi donare a'l mio cuor vita di Cino da Pistoia: cfr. l'introduzione di D De Robertis alla riproduzione anastatica dell'edizione giuntina: Sonetti e canzoni di diversi antichi autori toscani. I. Introduzione e indici, Firenze, Le Lettere, I977, p. 52 (il testo di Cino nel vol. II. Testo, c. 58 ).

i s. Arzocchi, Egloghe, p. 5. Nel commento a questi versi, p. i s, la Fornasiero per questo «artificio di citare nell'egloga l'incipit di un altro testo» rinvia alle bucoliche virgiliane (v 86 e 87 , e anche IX 24 e 27 , dove però troviamo non citazione ma 'traduzione' di Teocrito, un fenomeno non perfettamente assimilabile alla tradizione indiretta che qui ci interessa), all'altro caso arzocchiano già considerato di II I63-65, e a Boiardo, Pastorale v 25-26, per cui cfr. subito sotto nel testo.

i6. Arzocchi, Egloghe, p. 37.

17. Arzocchi, Egloghe, p. 43.

i 8. Matteo Maria Boiardo, Pastorale. Carte de triomphi, a cura di C. Montagnani e A. Tissoni Benvenuti, Novara, Interlinea, 2015 , p. 173.

19. Cristina Montagnani nel suo commento parla appunto di «prospettiva tutta e solo lirica», e anche per l'autocitazione arzocchiana osserva trattarsi di un «testo non noto, ma che potrebbe essere di registro lirico» (Matteo Maria Boiardo, Pastorale, p. 167).

20. Matteo Maria Boiardo, Amorum Libri tres, a cura di T. Zanato, Novara, Interlinea, 20I 2, p. 68.

2I. In questa autocitazione vedeva un punto critico F. Battera, La bucolica volgare del Boiardo, in «Interpres», VII (1987), pp. 7-44, a p. I 9: «Il fatto è apparentemente scontato e invece problematico: il codice bucolico prevede infatti che un pastore non ricordi il canto di colui al quale sono rivolti gli elogi e l'invito ad esibirsi: i pochi versi ricordati non vogliono essere un preciso rinvio ad opere effettivamente composte. Questo vale per Buc. IX 45 sgg. come pure per il Boiardo dei Pastoralia ... dove è chiaro che il Boiardo vuol solo imitare il luogo virgiliano corrispondente, non certo ricordare ai lettori i suoi precedenti tentativi bucolici. 
Ma non è così in Pastorale $\mathrm{V}$, dove Gorgo recita precisamente i primi due versi del primo sonetto degli Amorum libri. Tale infrazione del codice non sarebbe poi così significativa se Boiardo al posto del canzoniere avesse citato qualche verso dei Pastoralia ... Al contrario, far sì che Gorgo connetta la frottola che Menalca canterà all'esperienza lirica non può non essere segno di una certa consequenzialità cronologica».

22. Matteo Maria Boiardo, Pastoralia. Testo critico, commento e traduzione di S. Carrai, Padova, Antenore, i 996, p. 72 (a p. I 29 la traduzione: “Ti sarà sembrato forse di intonare qualcosa di bello quando «O rosa bella» fiorì col tuo canto $\mathrm{o}$ quando venne fuori, già noto perfino ai ragazzini, «Quel trionfo»").

23. Cfr. Carrai, che riporta nel cappello dell'egl. vII (ivi, p. 68), i vv. $63-65$ della III egloga di Tito Vespasiano Strozzi, «attribuiti dal pastore Albico [...] ad un Poeman che li canta nella terra di Gesso, facente parte del feudo boiardesco»: «Sic faciam, sed nec 'Rosa bella' nec 'Ille triumphus' / nunc mihi carmen erit, viridis quem frondea Gipsi / cantat apollineis nmphis per devia Poeman». Secondo lo studioso "Ille triumphus" potrebbe forse essere la latinizzazione dell'attacco incipitario del «capitolo ternario rivolto alla Vergine [...] Quel trionfo e la gloria di quel trono» (ibid.), ma su questo punto la ricerca dovrà essere approfondita.

24. Rapporti tra poesia e musica che costituiscono fra l'altro il tema centrale dell'egloga III delle Pastorale volgari dello stesso Boiardo: cfr. il cappello introduttivo di Cristina Montagnani in Matteo Maria Boiardo, Pastorale, pp. i $23-25$.

25. Cfr. G. Cattin, Canto, canzoni a ballo e danze nelle Maccheronee di Teofilo Folengo, in «Rivista Italiana di Musicologia», x, I 975 . In onore di Nino Pirrotta, pp. I 80-2 I 5, a p. 203; e l'edizione di Teofilo Folengo, Macaronee minori. Zanitonella - Moscheide Epigrammi, a cura di M. Zaggia, Torino, Einaudi, I987, p. 95.

26. Ai limitati fini specifici di questo contributo mi sono attenuto al testo edito da L. Frati, Un'egloga rusticale del I508, in "Giornale Storico della Letteratura Italiana», $\mathrm{Xx}, \mathrm{I} 892$, pp. I 86-204, a p. 200, ma certamente questo particolare componimento meriterebbe una edizione aggiornata e un adeguato commento.

27. Così li identifica il Frati, ma non è da escludere che «el ballettino» del v. I 83 vada sciolto in «e 'l ballettino», con ciò aumentando di una unità l'elenco. Non ho argomenti documentari per sostenere questa ipotesi, ma una osservazione testuale: da un altro testimone (nel Libro Septimo delle Frottole stampate da Ottaviano Petrucci nel I 507, nella cui edizione critica il testo si legge, con ricco commento, alle pp. IоI-I03: per i dati bibliografici cfr. la successiva nota 29) conosciamo l'incipit citato da Frati come Deh, levate la stringa dallo pecto: dunque è plausibile che il «De» iniziale valga come interiezione e non come preposizione, e perciò faccia parte del titolo, cosa che rende altrettanto plausibile che con «ballettino» si intenda un altro, diverso testo o ballo.

28. Cfr. Ruzante, Teatro. Prima edizione completa. Testo, traduzione a fronte e note a cura di L. Zorzi, Torino, Einaudi, I967, pp. 262-63 (testo) e p. I 335 (note). Un altro elenco simile, più breve, si trova nella scena III dell'atto II della commedia del mantovano Nicola Grasso, pubblicata a Venezia nel i 530 , Eutichia: cfr. l'edi- 
zione a cura di L. Stefani, Messina-Firenze, D’Anna, i978, p. 75. Ma un repertorio completo di queste presenze di canti (citati per lo più con l'incipit), nel teatro primocinquecentesco ancora manca.

29. Si tratta, in ordine cronologico di edizione critica, di: Frottole. Libro undecimo, Ottaviano Petrucci, Fossombrone, IsI4, edizione critica a cura di F. Luisi, edizione dei testi poetici di G. Zanovello, Padova, Cleup, I 997; Frottole. Libro octavo, Ottaviano Petrucci, Venezia, I507, edizione critica a cura di L. Boscolo, Padova, Cleup, I999; Frottole. Libro nono, Ottaviano Petrucci, Venezia, I508 (ma I509), edizione critica a cura di F. Facchin, edizione dei testi poetici di G. Zanovello, Padova, Cleup, I999; Frottole. Libro sexto, Ottaviano Petrucci, Venezia, Isos (ma I506), edizione critica a cura di A. Lovato, Padova, Cleup, 2006; Frottole. Libro septimo, Ottaviano Petrucci, Venezia, I507, edizione critica a cura di L. Boscolo, Padova, Cleup, 2006; Frottole. Libro primo, Ottaviano Petrucci, Venezia, Is04, edizione critica a cura di C. Di Zio Zanolli, Padova, Cleup, 201 3. Importanti osservazioni sulle prime tre di tali edizioni, ma aventi valore generale, in P. Trovato, In margine alle edizioni critiche del corpus petrucciano. Appunti linguistici, stilistici e metrici, in Venezia Isor: Petrucci e la stampa musicale. Venice Isor: Petrucci, Music, Print and Publishing. Atti del Convegno Internazionale Venezia - Palazzo Giustinian Lolin Io-I 3 ottobre 200I, a cura di G. Cattin e P. Dalla Vecchia, Venezia, Edizioni Fondazione Levi, 2005, pp. $253-76$, e in particolare alle pp. $262-63$ (a cui si rimanda anche per la bibliografia ivi registrata) per l'argomento qui trattato; argomento di cui si occupano, negli stessi Atti del convegno, anche l'intervento di F. Luisi, Scrittura e riscrittura del repertorio profano italiano nelle edizioni petrucciane, pp. I77-2 I4, alle pp. 195-97, e quello di R. Tibaldi, Repertorio tràdito e coevo nella intavolature per canto e liuto raccolte da Francesco Bossinensis con uno sguardo alle raccolte analoghe, pp. 49I-590, alle pp. 530-32, interventi ai quali pure si rimanda anche per la bibliografia pregressa.

30. Iacopo Sannazaro, Arcadia, introduzione e commento di C. Vecce, Roma, Carocci, 2013 , pp. $85-86$.

3i. Sannazaro, Arcadia, pp. $85-86$.

32. Cfr. ad es. in Publio Virgilio Marone, Le Bucoliche. Introduzione e commento di A. Cucchiarelli. Traduzione di A. Traina, Roma, Carocci, 2o r 2, il commento ai vv. 46-49, pp. 50I-502: «Con ogni probabilità Servio si riferisce in primo luogo ai vv. 46-49, che formano un gruppo compatto [...]; translati sarà da intendere non nel senso di una citazione letterale, ma più verosimilmente di un riadattamento». Però lo stesso Cucchiarelli si chiede poi se Virgilio non stia «davvero citando spezzoni di pentametri composti dall'amico» (p. 502), e poco sotto nota che uno degli emistichi «equivale alla metà di un pentametro (forse galliano?)».

33. Solo una ricerca esaustiva sull'onomastica dei 'pastori' della poesia bucolica potrebbe allineare degli eventuali precedenti di nome non travestito nell'ambito della produzione latina e volgare italiana. Sulla base dell'invecchiatissimo ma ancora indispensabile lavoro di E. Carrara, La poesia pastorale, Milano, Vallardi, I 905 , p. I 78 , andrà ricordato almeno il caso dell'egloga VII di Girolamo Benivieni, in cui uno dei cui interlocutori, Pico, sembra rispondere al nome reale (se si tratta di Giovanni Pico della Mirandola). Ma non mi pare dubbio che Sannazaro non abbia bisogno di mediazione e che qui il suo modello diretto sia il Gallo di Virgilio. 
34. Sannazaro, Arcadia, p. 247 e p. 256 (mia l'enfasi). E cfr. la sintesi dell'egloga nel commento di Vecce, p. 25 8: «Selvaggio, ricordando un suo esilio d'amore a Napoli, presenta la figura di un eccellente pastore udito laggiù, Caracciolo, e ne riferisce il canto: amaro atto d'accusa contro un duro presente, e profezia di una giustizia vendicatrice».

35. Sannazaro, Arcadia, p. 260 e p. 263.

36. Da M. Santagata, La lirica aragonese. Studi sulla poesia napoletana del secondo Quattrocento, Padova, Antenore, 1979, pp. 36r-64, al commento di Vecce nell'edizione cit., passando per M. Riccucci, Il neghittoso $e$ il fier connubbio. Storia e filologia nell'Arcadia di Jacopo Sannazaro, Liguori, Napoli, 200 I, in particolare alle pp. 35-38 e 94-97, e per E. Fenzi, Arcadia X-XII, in Travestimenti. Mondi immaginari nell'Europa delle corti, a cura di R. Girardi, Bari, Edizioni di Pagina, 2009, pp. 35-70, a p. 70.

37. Sannazaro, Arcadia, p. 263.

38. Le varianti della redazione A sono documentate nel commento di Vecce: $\mathrm{cfr}$. Sannazaro, Arcadia, pp. 250, 252, 254, 256.

39. Sannazaro, Arcadia, p. 247, col commento di Vecce: «Comincia il canto di Caracciolo [...]. Nel codice Vaticano [Vat. lat. 3202], in margine, la postilla: "Canto de Caracziolo"».

40. Cfr. Vela, Il Tirsi, cit., pp. 249-56.

4I. Il diverso parere di Giacomo Vagni, che in Baldassarre Castiglione Cesare Gonzaga, "Rime" e "Tirsi", assumendo le ipotesi di un suo precedente lavoro (G. Vagni, L'«honorata schiera» della duchessa Elisabetta. Ipotesi attributive per il «Tirsi» di Baldassar Castiglione e Cesare Gonzaga, in «Aevum», 87, 201 3, pp. 733-58) colloca Dolce e amaro destin tra le Rime dubbie del Castiglione, pp. 97-195, e Perché di lachrimar tra le Rime dubbie di Cesare Gonzaga, pp. I47-54 (e cfr. anche il commento ai rispettivi luoghi del Tirsi ove compaiono le citazioni, rispettivamente a p. 239 e a p. 243 dell'edizione), non poggia secondo me su argomentazioni stringenti: in assenza di prove certe, continua a sembrarmi che il grado di probabilità' delle attribuzioni sia più favorevole a Muzzarelli e Pallavicino che a Castiglione e Gonzaga. 